\title{
Mejoras en un centro de distribución mediante la simulación de eventos discretos
}

\section{RESUMEN}

En la presente investigación se elaboró un modelo de simulación para proponer y evaluar mejoras en un centro de distribución de alimentos de consumo humano. Se tomaron como indicadores para evaluar el desempeño del sistema; el nivel de servicio que presta el centro, el tiempo de respuesta, tanto en la recepción como el despacho y los costos operativos. Con el modelo se ejecutaron una serie de experimentos; como la distribución en planta del almacén, modificaciones en los procesos de recepción y despacho, obteniendo así una configuración que aumenta el rendimiento del sistema en estudio en aproximadamente un $40 \%$.

Palabras clave: centro de distribución, distribución en planta, simulación de eventos discretos

IMPROVED DISTRIBUTION CENTER BY DISCRETE EVENT SIMULATION

\section{ABSTRACT}

In the present investigation a simulation model was elaborated to propose and to evaluate improvements in a center of distribution of foods of human consumption. They took as indicators to evaluate the acting of the system; the level of service that perform the center, the time of answer, so much in the reception as the despatch and the operative costs. With the simulation model they were executed a series of experiments; as the plant layout of the warehouse, modifications in the reception processes and shipping, obtaining this way a configuration that the yield of the system increases in study in approximately $40 \%$.

Keywords: distribution center, plant layout, simulation of discreet events, warehouse

\section{INTRODUCCIÓN}

La gestión de almacenamiento es un eslabón esencial en la cadena de suministro; que planifica, implementa y controla la recepción, resguardo y custodia de los materiales, desde los puntos de abastecimientos a través de sus proveedores, hasta el despacho de los productos terminados a los clientes, teniendo como objetivo prestar el nivel de servicio requerido al menor costo posible. Las actividades de almacenamiento se han considerado como una actividad sin valor agregado, por lo tanto, un desperdicio desde el punto de vista de los criterios de manufactura esbelta, por lo tanto se considera una carga costosa para la cadena de suministro, sin tomar en cuenta aspectos cualitativos de otras funciones de la misma. Pero este criterio ha venido cambiando, en la actualidad las nuevas tecnologías y las novedades técnicas de mercadeo y la integración de la cadena de suministros, ha provocado mayor interés de las empresas, en la logística como elemento de análisis y mejoras continuas, lo que ha provocado que se busquen técnicas para obtener mejores resultados y utilizarlos como elementos de competitividad, al mejorar los niveles de servicio al cliente.

En un almacén se desarrollan actividades que van desde la recepción de la mercancía hasta su despacho, pasando por el resguardo de la misma, la preparación de pedidos, control de los inventarios; todo esto hace que ésta sea una tarea muy compleja para ser estudiada a profundidad por métodos tradicionales. La simulación viene a ser una herramienta ideal para estudiar sistemas complejos, evaluando diferentes alternativas con su respectivo análisis de sensibilidad a un costo relativamente menor que otras técnicas, pudiendo tomar la mejor decisión sin necesidad de alterar previamente el sistema.

En la presente investigación se utilizó la simulación de eventos discretos como herramienta para proponer mejoras en un centro de distribución, en lo que respecta a las actividades de recepción, almacenamiento y despacho, obteniendo la configuración para prestar el servicio de una forma adecuada al menor costo posible.

\footnotetext{
Departamento de Investigación Operativa. Facultad de Ingeniería, Universidad de Carabobo, Valencia, Venezuela

** Departamento de Ingeniería de Métodos. Facultad de Ingeniería, Universidad de Carabobo, Valencia, Venezuela.
} 


\section{ALMACENAMIENTO}

Thomkins et al. (2011) señala que a media que el tiempo transcurre aparecen nuevos y más complejos procesos de almacenamiento y por lo tanto se requiere el uso de técnicas de planificación de estas actividades, entre los problemas más comunes está la organización de las áreas de almacenamiento, la minimización de los recorridos, clasificación de los materiales, uso correcto de los equipos de manejo de materiales, existen técnicas cuantitativas para mejorar estas actividades pero son de difícil aplicación, por lo tanto otra forma de enfocar eficientemente estos problemas, es la aplicación de técnicas de simulación de tales procesos, con resultados favorables y productivos a la hora de tomar decisiones .

Quiroga (2009) Menciona que los principales objetivos del almacenamiento son: aprovechar el espacio total, lograr un fácil acceso a los materiales, conseguir la mínima manipulación de la mercancía almacenada, facilitar la rotación y el control del inventario. Señala, además, que las funciones de un almacén son: amortiguar el desequilibrio que se presenta entre oferta y demanda, disminuir costos y servir como complemento al proceso de producción.

Carranza y Sabriá (2005) definen el almacenamiento como aquellas actividades que permiten el adecuado almacenaje de productos y materiales, así como la preparación de pedidos, indicando además, que se considera como una actividad que no agrega valor al producto; pero que su importancia radica en tener el producto o los materiales en el momento oportuno, en el lugar correcto y en las cantidades requeridas.

\section{SIMULACIÓN}

García et al. (2006) definen la simulación como el conjunto de relaciones lógicas, matemáticas y probabilísticas que integran el comportamiento de un sistema, siendo su objetivo lograr comprender, analizar y mejorar las condiciones de operación de dicho sistema. La importancia de la simulación como herramienta para resolver problemas radica en su potencial en el estudio de sistemas en los cuales la solución analítica no existe o es muy difícil de obtener.

Se dice que la simulación es de eventos discretos cuando se emplea para el análisis de sistemas en lo que su estado cambia en puntos separados de tiempo debido a la ocurrencia de un evento; tal como sucede en el caso de estudio.
Zhou et al. (2004 y 2005) realizaron investigaciones en las que elaboraron modelos de simulación para el estudio de almacenes, donde representaron los procesos operativos claves de estos sistemas (recepción, ubicación, preparación y despacho de un pedido), concluyendo que la simulación es una herramienta poderosa que ayuda a los analistas a tomar buenas decisiones en el análisis de este tipo de sistemas.

Gagliardi et al. (2007) modelaron las diferentes actividades que se ejecutan en un centro de distribución, planteándose como objetivo evaluar las estrategias empleadas en la preparación y embalaje de un pedido, así como el espacio destinado para el resguardo de los diferentes productos buscando minimizar los costos de operación, manteniendo el nivel de servicio exigido por el cliente. Takakuwa et al. (2000) estudió por medio de esta herramienta el manejo de materiales, analizando la recepción, el despacho y la ubicación de las distintas mercancías.

Magableh y Rossetti (2005) desarrollaron un modelo genérico de simulación para representar las operaciones de un centro de distribución, específicamente en la recepción de productos y despacho de pedidos, considerando la cantidad de recursos disponibles, el uso del modelo está dirigido a los planificadores, con el propósito que se pueda estudiar el comportamiento del sistema ante cambios en la demanda y así tomar las medidas preventivas para garantizar el adecuado funcionamiento del mismo.

\section{CASO DE ESTUDIO}

\subsection{El Sistema}

El sistema en estudio es un centro de distribución de alimentos de consumo humano en el cual se reciben materias primas desde el exterior y productos terminados desde tres plantas ubicadas en el interior del país. Así mismo se despachan los productos terminados recibidos de dichas plantas hacia tres plataformas de "crossdocking" y dos centros de distribución para que estos concluyan la fase de distribución de los productos hasta los mayoristas y minoristas

El almacén maneja un total de 8 familias de productos: A, B, C, D, E, F, G y Materias Primas, consta de $5.022 \mathrm{~m}^{2}$ en la cual se contabilizan 6.700 posiciones en 7 niveles de altura con un total de 8 puertas para 7 pasillos, donde 4 son para despacho y 4 para recepción. Cada pasillo tiene $3,15 \mathrm{~m}$ de ancho, las puertas tienen un ancho de 2,31 m. (ver figura 1). 
Figura 1. Distribución del almacén.

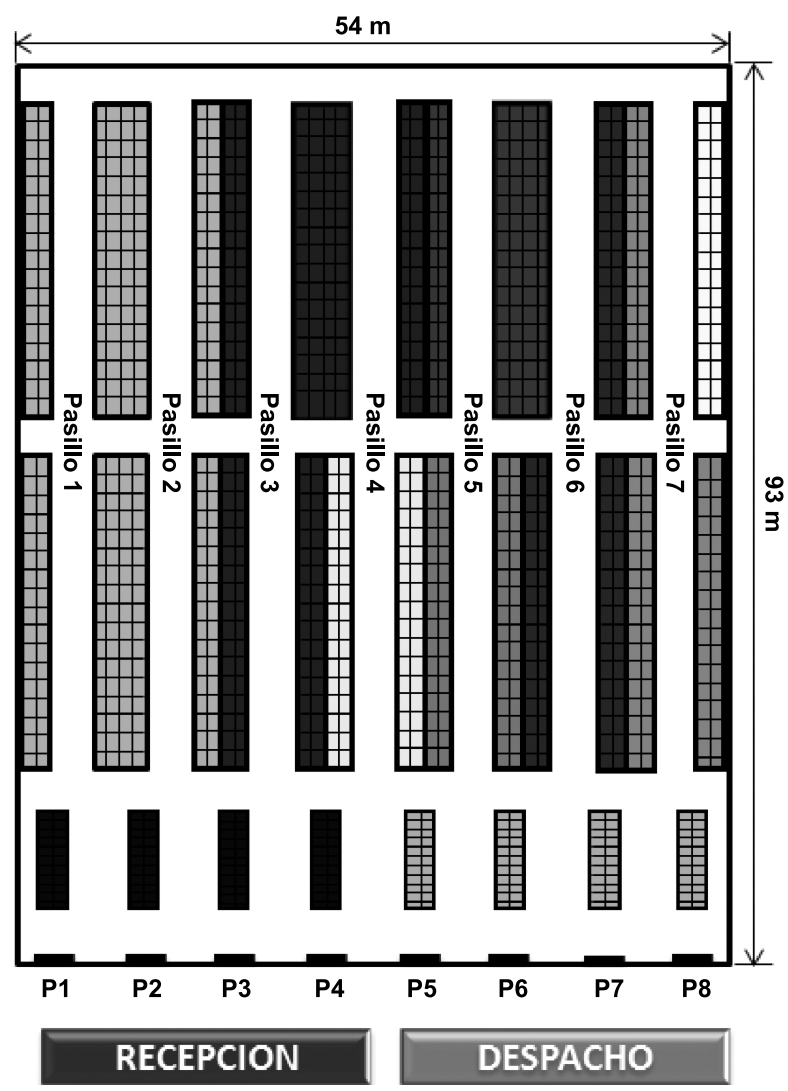

La zona de descarga es el área donde se ubican las paletas que se reciben existiendo una para cada puerta de recepción, posee un área de $39 \mathrm{~m}^{2}$ al igual que las zonas de carga, pertenecientes a cada puerta de despacho, donde son ubicadas las paletas que se preparan para una orden de pedido.

Posee cuatro montacargas eléctricos, y cinco transpaletas como equipos móviles con sus respectivos operadores. La jornada laboral está dividida en dos turnos de ocho horas con una interrupción de una hora para comer.

\section{PROCESOS DEL CENTRO DE DISTRIBUCIÓN}

\subsection{Proceso de Recepción}

El proceso de recepción se inicia con la llegada de los camiones de los proveedores, inmediatamente se procede a bajar las paletas de productos, ubicándolas en la zona de descarga, esta actividad es realizada por un operador con una transpaleta, una vez ubicada toda la carga, se realiza un chequeo para verificar el tipo y la cantidad de productos que se reciben, luego se registra en el sistema y designándose su ubicación en el almacén, la cual es realizada por un montacarguista llevándolos hasta cada una de las locaciones determinadas para los productos.

\subsection{Proceso de despacho}

Se inicia con la llegada de las órdenes de despacho de acuerdo al volumen de carga se define el número total de camiones necesarios, tomando en cuenta la la capacidad de carga de los camiones y la cantidad de paletas de productos demandados por cada cliente. Se hace la preparación del pedido y se procede a buscar los productos que corresponden a la orden, desde su locación hasta la zona de carga. Sí la orden requiere de la preparación de los pedidos (picking), la realiza un operador con una transpaleta, extrayendo cantidades más pequeñas de materiales de las paletas a cajas, para luego ubicarlas en la zona de carga. Una vez preparada la orden por camión, se realiza la verificación de acuerdo a la solicitud del cliente según tipo y cantidad de productos, luego se procede a la cargar el camión con una transpaleta, y se despacha.

Lo que se busca es aumentar el desempeño del centro de distribución, específicamente aumentando nivel de servicio, y disminuyendo el tiempo de respuesta de los procesos de recepción y despacho.

El nivel de servicio de recepción y despacho es considerado como el porcentaje de camiones que son atendidos de inmediato. El tiempo de respuesta para el proceso de recepción se contabiliza desde que llega un camión hasta que es cargado, mientras que para el despacho se mide desde que se empieza a tramitar una orden hasta que la misma es despachada.

\section{EL MODELO}

A continuación se muestran las actividades a simular, las suposiciones consideradas para elaborar el modelo de simulación, y las variables y restricciones. Se usa el programa de simulación ARENA $₫$ y la representación animada del modelo se muestra en la figura 2.

\subsection{Actividades a Simular}

Llegada de camiones de recepción: Indica el número de camiones que llega con productos para almacenar en el centro de distribución desde las tres plantas de las cuales se reciben productos, así como los que llegan del exterior. En esta actividad también se simula las cantidades recibidas de cada familia de producto. 
Descarga: Representa la operación de la descarga de cada una de las paletas que contienen los camiones que llegan al almacén, tiene un tiempo asociado, así como una cantidad de viajes realizados con los transpaletas desde el camión hasta la zona de descarga, que se determinan según la cantidad de paletas que se estén descargando.

Ubicación: Una vez descargadas las paletas de los camiones, los montacarguistas proceden a ubicarlas en los racks correspondiente según la familia a la que pertenezca el producto, esto se simula tomando en cuenta el tiempo que se toma hacer las rutas desde las zonas de descarga hasta la ubicación de los distintos productos.

Llegada de órdenes: Son las órdenes que llegan diariamente a las plataformas crossdocking, transferencia o solicitud de materias primas, trayendo la información sobre la cantidad de paletas demandadas por familia de productos.

Preparación de órdenes: Representa la búsqueda de los productos para la elaboración de las órdenes requeridas. Se toma en cuenta para esta actividad los tiempos de recorrido desde la zona de descarga hasta la ubicación de los productos que requiere la orden, así como la cantidad demandada de cada uno de los productos, la cual determina el número de viajes que realizará el operario para preparar la orden.

Picking: en esta actividad se recolectan con un transpaleta los productos que requiera una orden en una unidad menor al de una paleta. Los productos, envasados en cajas, son colocados en una paleta por un operador, el cual realiza el recorrido por las áreas donde se encuentran los distintos productos que posea una familia, hasta completar la capacidad de una paleta y luego procede a buscar otra, para continuar recolectando las cajas de productos que se necesiten para completar una orden. Se consideran los tiempos para hacer los recorridos desde las zonas de carga hasta la zona de cada familia de productos, el tiempo que demore el operador en desconsolidar y extraer de la paleta el producto necesario, así como también la cantidad de paletas que se formarán con los requerimientos de cada orden.

Llegada de camiones a despachar: Esta actividad considera la llegada de los camiones que provienen desde las 3 plataformas de crossdocking, de los dos almacenes y de la planta de producción, que llegan a cargar las órdenes.

Carga de camiones: Se considera el tiempo asociado al recorrido que hace un montacarguista desde la zona de carga hasta la puerta donde se encuentra el camión, así como la cantidad de viajes que deba realizar el mismo para completar la carga del camión.

\subsection{Supuestos a Considerar}

Siempre hay existencia de productos en inventario.

Los recorridos en el almacén se hacen hasta el punto medio del pasillo donde se encuentra la ubicación de la familia de productos de interés.

Los recorridos de los montacarguistas para transportar productos en el almacén se realizan siempre con 2 paletas por viaje.

\subsection{Variables de Entrada}

Número de camiones que llegan desde cada una de las plantas.

Tiempo de descarga de un camión.

Tiempo de carga de un camión.

Tiempo de recorrido desde cada zona de descarga hasta cada una de la familia de productos para la ubicación de los mismos.

Tiempo de recorrido desde cada familia de productos hasta cada zona de carga para la preparación de los pedidos.

Demanda de los productos

\section{RESULTADOS}

Se realizaron una serie de experimentos para evaluar comportamiento actual del sistema obteniéndose los resultados que se muestran en la tabla 1.

En el centro de distribución se observó un arreglo ineficiente de las áreas de almacenamiento debido a que los productos más demandados están lejos de la zona de recepción y despacho, ocasionando largos recorridos y un aumento considerable en los tiempos de operación, adicionalmente el $87 \%$ del tiempo están ocupadas las zonas de despacho mientras que solo un $34 \%$ las de recepción, también se observaron vehículos esperando por ser despachados, ya que deben esperar no solo a que se desocupe la zona de carga, sino que además deben estar disponibles los operadores, todo esto ocasiona que los tiempos de ubicación de los productos y preparación de las órdenes sea en promedio de 2,75 y 5 horas respectivamente.

Se realizó una redistribución de las áreas de almacenamiento, colocando las familias de productos más demandados en ubicaciones cercanas a las zonas de despacho, además 
Figura 2. Representación animada del modelo en ARENA®.

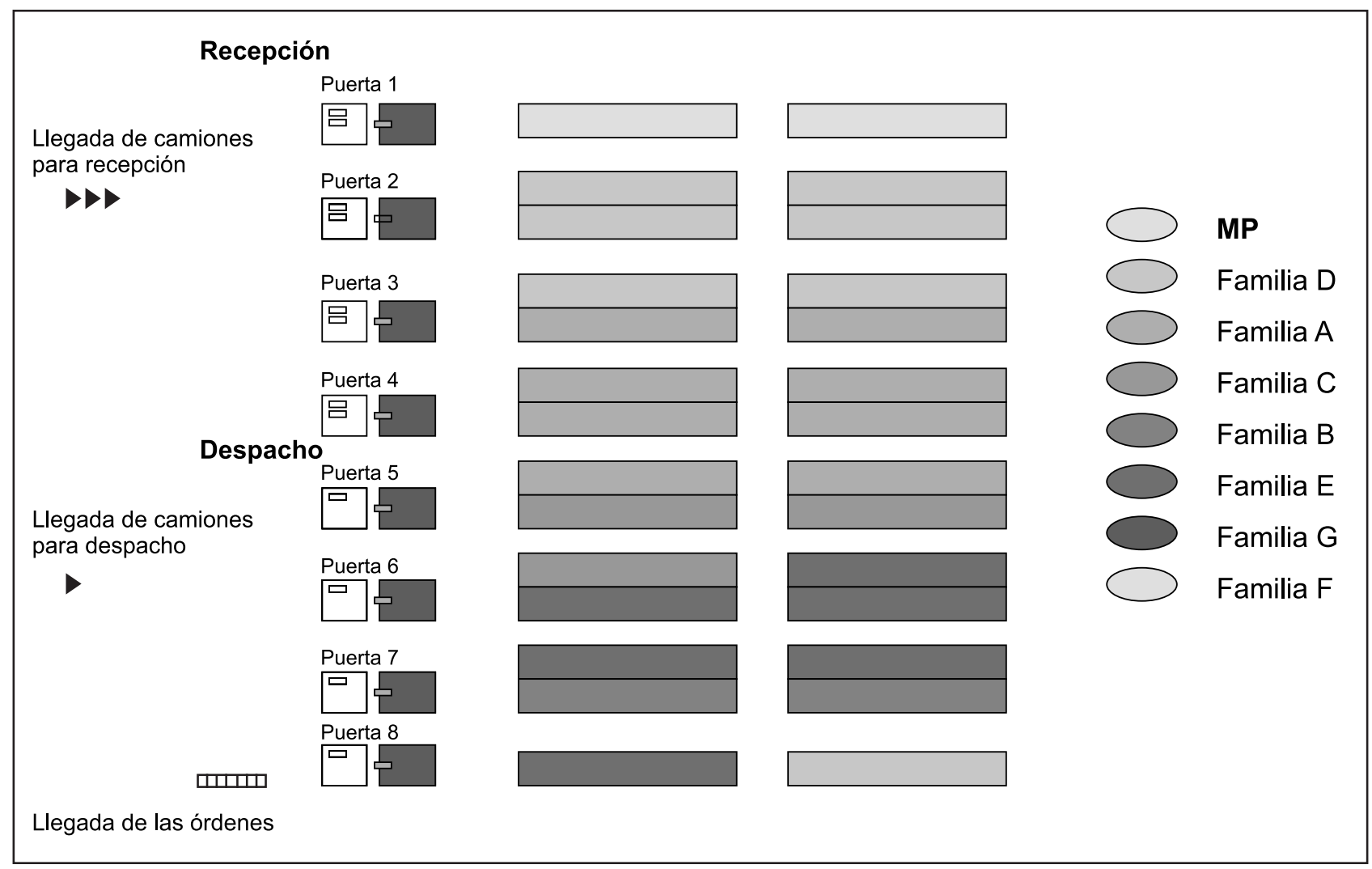

Tabla 1. Valores de los indicadores para el sistema actual

\begin{tabular}{|c|c|}
\hline Indicadores & Sistema \\
\hline Nivel de Servicio de Recepción (\%) & $56,27 \pm 1,16$ \\
\hline Nivel de Servicio de Despacho (\%) & $55,89 \pm 1,07$ \\
\hline Tiempo de respuesta de recepción (hrs) & $2,75 \pm 0.02$ \\
\hline Tiempo de respuesta de despacho (hrs) & $4.99 \pm 0,47$ \\
\hline
\end{tabular}

Fuente: ARENA®.

se destinó una puerta de descarga para carga, producto de que la utilización de estas últimas es considerablemente mayor ( $87 \%$ contra $34 \%$ ) y se duplicó el área destinada a cada una de las zonas de carga de manera tal de tener en simultáneo la cantidad de paletas equivalente a lo requerido por dos camiones, de forma tal continuar con la preparación de órdenes, sin esperar que este libre alguna de estas zonas, evitando así que se detenga el proceso de despacho.

Al simular los cambios sugeridos se obtienen los resultados presentados en la tabla 2 , donde se aprecia un incremento considerable en el nivel de servicio y una disminución en el tiempo de respuesta sobretodo del despacho.

\section{CONCLUSIONES}

Las actividades de almacenamiento juegan un papel importante en la cadena de suministro de cualquier empresa y no agrega valor de uso al producto, y aunque se considera una carga costosa, las empresas para poder sobrevivir en un mercado competitivo, han puesto énfasis en mejorar estas actividades con el empleo de técnicas cuantitativas. Con el avance técnico de los últimos años, se han realizado investigaciones en las que se elaboraron modelos de simulación para el estudio de almacenes, donde se representan procesos operativos claves de estos sistemas (recepción, ubicación, preparación y despacho de pedidos), 
Tabla 2. Valores de los indicadores con la propuesta.

\begin{tabular}{|c|c|}
\hline Indicadores & Sistema \\
\hline Nivel de Servicio de Recepción (\%) & $68,42 \pm 1,005$ \\
\hline Nivel de Servicio de Despacho (\%) & $89,65 \pm 0,668$ \\
\hline Tiempo de respuesta de recepción (hrs) & $2,57 \pm 0,05$ \\
\hline Tiempo de respuesta de despacho (hrs) & $4,06 \pm 0,25$ \\
\hline
\end{tabular}

Fuente: ARENA®.

obteniéndose resultados prácticos y económicos respecto a otras de difícil y costosa aplicación.

La gran fortaleza de la herramienta utilizada, $A R E N A \circledast$, es que una vez que se ha construido el modelo que representa a la situación actual es muy sencillo probar diferentes alternativas sin poner en riesgo al sistema, donde cualquier persona con conocimientos básicos de simulación puede probar diferentes alternativas o realizar los análisis de sensibilidad deseado, cuestión que no se puede lograr con los métodos cuantitativos tradicionales. y estimando el desempeño del almacén en segundos.

El aporte de esta investigación es la producción del modelo particular que representa fielmente a la empresa estudiada, éste aplicable con pocas modificaciones a empresas o situaciones análogas, con un tiempo de procesamiento casi instantáneo, lo que permite rápida respuesta y adaptación de los sistemas de manejo de materiales, generando un modelo dinámico de mejora continua.

El estudio fue realizado en un centro de distribución de alimentos de consumo humano, en el cual se tomaron en cuenta las variables de entrada y salida que definen el sistema de recepción, custodia y despacho, con la construcción del modelo y la aplicación del programa, éste probó ser una herramienta de análisis, precisa y útil para la toma de decisiones, sin incurrir en costos adicionales en el futuro.

Del análisis previo se observó la existencia de un arreglo ineficiente de las áreas de almacenamiento, evidenciado en que los productos de mayor demanda están lejos de la zona de recepción y despacho, originando largos recorridos y un aumento considerable en los tiempos de operación, además el $87 \%$ del tiempo estaban ocupadas las zonas de despacho mientras que solo un $34 \%$ las de recepción.

De los diversos experimentos realizados con la herramienta ARENA $®$, se obtuvieron los siguientes resultados: el Nivel de Servicio de Recepción pasó de $56,27 \pm 1,16$ a $68,42 \pm 1,005$ se incrementó en $21,59 \%$, el Nivel de Servicio de Despacho (\%) de 55,89 a 89,65 aumento del $60,4 \%$, el Tiempo de respuesta de recepción (hrs) de 2,75 a 2,57 disminuyó en $6,5 \%$ y el tiempo de respuesta de despacho (hrs) de 4,99 a 4,06 con una disminución del $3,7 \%$.

\section{REFERENCIAS BIBLIOGRÁFICAS}

[1] Carranza, O. y Sabriá, F. (2005). Logística. Mejores prácticas en Latinoamérica. Thomson.

[2] Gagliardi, J., Renaud, J. y Ruiz, A. (2007). A simulation model to improve warehouse operations. Winter Simulation Conference ( $p$. 2012-2018).

[3] García, E., García, H. y Cárdenas, L. (2006). Simulación y análisis de sistemas con ProModel. Pearson Prentice Hall.

[4] Magableh, G. y Rossetti, M. (2005). Modeling and analysis of a generic cross-docking facility. Winter Simulation Conference (p. 1613-1620).

[5] Quiroga, J. (2009). Logística de entrada. Abastecimiento - logística de compra transporte - stocks - diseño de almacenes.

[6] Takakuwa, S., Ito, K., Takizawa, H. y Hiraoka, S. (2000). Simulation and analysis of nonautomated distribution warehouse. Winter Simulation Conference (p.1177-1184

[7] Tompkins, J., White J., Bozer Y,Tanchoco J., (2011). Planeación de Instalaciones. Thomson Learning. Cuarta edición, Mexico D. F.

[8] Zhou, M. Son, Y. y Chen, Z. (2004). Knowledge representation for conceptual simulation modeling. Winter Simulation Conference $(p$. 450-458).

[9] Zhou, M. Setavoraphan, K. y Chen, Z. (2005). Conceptual simulation modeling of warehousing operations. Winter Simulation Conference ( $p$. 1621 - 1626). 